\title{
Large-scale doming on Europa: a model of formation of Thera Macula
}

\author{
Loïc Mével* and Eric Mercier*
}

*Laboratoire de Planétologie et de Géodynamique (UMR-CNRS 6112)

Faculté des Sciences et Techniques / 2, chemin de la Houssinière / BP 92208 / 44322 Nantes cédex 3.

loic.mevel@nantes.fr

Eric.Mercier@univ-nantes.fr

25 pages, 9 figures, 2 tables

Correspondence to: Loïc Mével (loic.mevel@nantes.fr)

Laboratoire de Planétologie et de Géodynamique (UMR-CNRS 6112)

Faculté des Sciences de Nantes

2, chemin de la Houssinière - BP 92208

44322 Nantes cédex 2 - France

\begin{abstract}
Since Galileo spacecraft reveals Europa's surface at high and medium resolution, deformation and processes affecting the relatively young surface have been more accurately defined. This work reports the observations carried out on a large-scale feature of the south hemisphere, Thera Macula. It is shown that Thera presents common points with many other features including small-scale domes, lenticulae and large-scale chaotic area (disrupted ancient surfaces lying on a dark matrix), but remains singular through its asymmetric morphology. On the basis of observations, we propose a scenario for the setting of Thera Macula. It involves a large-scale doming (40-70 km in radius) of the pre-existing surface associated to ductile deformations, and the consecutive collapse of the created megadome associated to brittle disruption of blocks and flow of low viscosity material over the surrounding ridged plains. The processes responsible for each stage of the proposed scenario have been investigated. Both cryomagmatic and diapiric origins are discussed and confronted to observations. Finally, comparison of similar features at various scales suggests that Thera Macula by its originalities (asymmetry, rounding bulge) may have preserved the intermediate stages of the formation of subcircular chaos at least up to about $50 \mathrm{~km}$ in radius. A common evolution and endogenic origin for multi-scale hot spot features is proposed: 1) the doming stage, 2) the collapse and extrusion stage, and 3) the relaxation stage.
\end{abstract}




\section{Introduction}

Europa's surface is one of the smoothest among the solar system bodies. However, Voyager and more recently Galileo's images reveal a surface widely disturbed by "geological" features (Lucchitta and Soderblom, 1982; Greeley et al., 2000; Figueredo and Greeley, 2000). These observations suggest that processes have driven movements in the icy crust in the past and possibly recently and have refreshed the surface (Pappalardo et al., 1998; 1999; Mével and Mercier, 2005). This last point is supported by the lack of impact craters compared to Ganymede and Callisto implying a young mean surface age ranging from 30 to $70 \mathrm{Myr}$ (Zahnle et al., 2003). The heat transfer from the silicate interior up to the icy surface may be a driven mechanism to explain dynamics of the icy crust (e.g. Cassen et al., 1979; Reynolds and Cassen, 1979; Ojakangas and Stevenson, 1989; Pappalardo et al., 1998; McKinnon, 1999; Thomson and Delaney, 2001). Furthermore, jovian tides generate friction leading to a substantial increase of heat in the icy crust. This may preserve an ocean under the ice (e.g. Pappalardo et al., 1999; Tobie et al., 2003).

Understanding of both the "geological" processes and the icy crust structure is indissociable from the quantification of the thermal regime in order to appreciate the dynamics of Europa. For this reason, we have studied the morphology of a feature named Thera Macula by using Galileo's SSI images obtained at $\sim 300 \mathrm{~m}^{\text {.pixel }}{ }^{-1}$ during the E17 orbit. This subcircular structure (140 by $80 \mathrm{~km}$ in diameter) associated with a similar but longer feature, Thrace Macula, is located in the south hemisphere (centred at $\sim 47.7^{\circ} \mathrm{S}$ and $180.9^{\circ} \mathrm{W}$ ). They were previously described and assumed to be cryomagmatic in origin by analyzing the lower resolution images acquired by Voyager imaging system (Wilson et al., 1997). On the basis of the morphological observations of Galileo images presented in section 2, we propose a plausible model of formation of Thera Macula. Our model suggests large-scale vertical deformations (i.e. doming) and its collapse including extrusion of subsurface material, similar to those observed at small-scale on domes and lenticulae (Pappalardo et al., 1998; Greenberg et al., 2003). In section 4, we will investigate two possible end-members for icy crust dynamics leading to the formation of Thera Macula, and compare Thera to other features similar to its size on Europa. Finally, we will discuss about "geological" processes and propose a possible genetic relation between chaotic areas in agreement with our model of evolution of Thera Macula.

\section{Geological settings}

From a stratigraphic point of view, the younger feature in a given area must be entirely preserved and not disturbed by any other feature except those resulting from exogenic or surface processes (i.e. impact craters, erosion, mass wasting). According to this principle, Thera Macula is young in the considered region and probably in the Europa's geological history. The lack of secondary impact craters on the surrounding ridged plains is a strong argument to exclude a meteoritic origin for Thera Macula.

We firstly distinguish two different areas on Thera Macula. One is bright ( $t b$ on Fig. 1), has a texture similar to ridged plains units and spreads on about 3500 to $4000 \mathrm{~km}^{2}$. The other is dark and rugged ( $r m$ on Fig. 1), and spreads on about $5000 \mathrm{~km}^{2}$. The limit between both units is relatively irregular but cuts Thera Macula almost through its central part.

\subsection{The northern bright area}

Topography, albedo and texture of the NW area appear relatively smooth and similar to ancient ridged plains units. The preexisting linear features cutting across this area are weakly disturbed, but several blocks appear to have been tilted and sometimes rotated and 
partially destroyed around the south boundary of bright unit (Fig. 2e, f). In general, deformation remains weak, although it becomes stronger toward the south (see Fig. 2a). Both brittle and ductile strains are visible on this area. However, whereas brittle deformation seems to predominate in the centre, boundaries of the bright unit are characterized by deformation fronts looking like ductile deformations. These fronts form a fracture network at the top of a linear bulge rounding Thera Macula suggesting the collapse of the adjacent ridged terrains toward the centre of Thera Macula (Fig. 2b, c, d). Fractures disappear to the west but the bulge always exists. The west and northeast bulges converge at the northwest tip of Thera but never joint together.

At the centre of Thera Macula, we can observe disturbed bright terrains. In places, some scarps have morphologies looking like "cuesta": a gentle E-W slope breaking off by a cliff from tens to hundreds meters in height (Fig. 2a, e) as indicated by the shadows and by using the simple equation:

$$
h=\frac{l \cdot \cos \left(\alpha_{i}\right) \cdot \cos \left(\alpha_{e}\right)}{\sin \left(\alpha_{e}+\alpha_{i}\right)}
$$

where $h$ is the height of the scarp, $l$ is the shadow length under solar incidence and emission angle respectively $\alpha_{l}$ and $\alpha_{e}$ (in degrees) with respect to the normal axis to the Europa's surface. Values of some significant scarps derived from this method are given above images in figure 2. These features seem to draw the borders of blocks tilted during Thera's emplacement toward the centre of Thera Macula over the chaotic rugged, dark and weak material $\mathrm{rm}$ seen on Fig. 1 to the south of Thera Macula. This last observation suggests that this unit represents a part of Thera Macula, which has undergone the same "tectonic" history than the southern chaotic unit.

\subsection{The southern chaotic area}

The southern terrains of Thera Macula are low albedo rugged units very similar to the chaotic terrains found on Conamara Chaos (e.g. Collins et al., 2000). Blocks of various sizes are elevated over the dark matrix and have been moved and sometimes tilted. The largest visible blocks are located close to the northern bright unit boundary and seem to come from it before they rotate, tilt and partially sink in the matrix material (Fig. 2f, g). Where it was possible, their heights have been estimated by using the shadow measurement and equation 2.1. (see table 1) in order to give some relative landmarks, but uncertainties remain important (i.e. one pixel gives an error ranging from 72 to 74 metres on the values of the height). The matrix material is generally confined under the ridged plains level as well as the northern bright unit by large cliffs, except the south border where the matrix material overflows the bright ridged plains forming a large cliff of few hundreds meters in height (about 437 metres, see Fig. 2h).

Finally, dark diffuse patches are observed in the ancient ridged plains along the east and south borders of Thera Macula (dt on Fig. 1).

\section{Implications for the observations}

\subsection{Doming of the surface at large scale}

As seen on the Fig. 1, morphologies of both northeast and west borders of Thera Macula are relatively similar: concentric bulges round bright and partly chaotic areas of Thera. The NE border is surrounding by one or two fractures resulting from the collapse of multi-kilometric blocks of pre-existing ridged terrains toward the centre of Thera Macula. 
These faults propagate to the NW at the top of the concentric bulge of a few kilometres width. However, the west border doesn't exhibit any associated fractures and the bulge system tends to disappear progressively southward. Finally, the matrix material overflows the pre-existing terrains to form a cliff at the south tip of Thera (Fig. 2h). Consequently, this cliff should not represent its original limit. Since the boundary seems to be defined by a N-S elongated ellipsoid elsewhere (e.g. the concentric bulge), we propose to fit and close the concentric bulge to the south (Fig. 3). This supposed original shape approximately describes an ellipsoid which radius ranges from 40 to $70 \mathrm{~km}$. We conclude that these concentric bulges and their associated fractures are relics of the periphery of a megadome extending over Thera Macula before its collapse. As explained in figure 4, uplift by punching of the pre-existing surface generates a stretch on the borders of the punched area (i.e. ductile deformations). This leads to a local crustal thinning and surface excess marked by the concentric bulge. The growth of the megadome tends to accumulate stresses near its top. Consequently, tensile cracks appear and dismantle the pre-existing surface over Thera Macula area more strongly in the south (chaotic area) than in the north (bright area). In this case, the strong asymmetry results from the gravity collapse of blocks toward the south, whereas the northern area remains more or less well preserved (Fig. 5c). The morphological discrepancies within the concentric bulge system and between the both units may be significant of a response variation of the superficial crustal material during the collapse (e.g. due to a difference in the topographic level between NW and SE of Thera Macula or due to lateral variation of the mechanical properties of the icy crust). At the end of the doming stage, positive topographic anomaly as large as Thera Macula becomes gravitary unstable and collapses.

\subsection{Collapse of the megadome}

As seen in the previous sections, the growth of a megadome more than $50 \mathrm{~km}$ in radius is strongly inferred by observations. Nevertheless, current morphology of Thera Macula implies, consecutively to the doming stage, a collapse that should be successively controlled by at least three processes.

\subsubsection{Gravity-controlled collapse}

After the doming stage (Fig. 5b), the rigid upper part of the icy crust tends to accumulate tensile stresses near the top of the maximal inflexion, leading to its failure in places. The strong asymmetry of Thera Macula reflects preferential sliding toward the south. In the same way as extensional or post-orogenic processes on Earth, main associated fractures over the south part of the megadome (i.e. current chaotic part of Thera Macula) may propagate down to a main shallow décollement zone and may define tilted blocks sliding southward up to the total collapse of the megadome (Fig. 5c-d). Although there is not any observation indicative of blocks coherently tilted on Europa, this way of collapse should not be excluded and especially in region associated with cryomagmatism where the surface is renewed and the icy crust partially destroyed.

The amount of extension should be partially compensated by a compression area in south front of Thera Macula. The mass loss due to blocks sliding combined to the hot thermal context at Thera's place could lead to thinning and gravity instabilities of the megadome facilitating its collapse. Gravity forces coupled to thermal weakening are needed to explain the dichotomy between northern preserved and southern chaotic terrains.

\subsubsection{Flows during the collapse}

Gravity processes alone do not explain all observations like the flow of material inside the chaotic matrix and over the south border of Thera Macula. Following the uplift of the 
megadome, sufficiently fluid material rises under pressure from beneath up to the surface along the major cracks in the rigid upper crust. Then, this fluid material is expected to spread on the surface during the collapse of the megadome (Fig. 5c). Numerous melting blocks are engulfed and dragged along the flowing direction (Fig. 5c-d) and the space between tilted blocks should then be filled by fluid matrix material. This mechanism requires the existence of viscous material sufficiently fluid to spread relatively quickly on the ground (i.e. slushy material). Besides, the presence of two diffuse patches on the south and east borders of Thera Macula ( $d t$ in Fig. 1) could agree with infiltration of fluid material, probably brines or slurries, coming from the matrix according to the interpretation given by literature about the same patches along the neighbouring feature Thrace Macula (Wilson et al., 1997; Kortz et al., 2000; Fagents, 2003).

Although this cryovolcanic event is shown on the last sketches of Fig. 5e, it might occur early during the gravity-controlled collapse. In this case, the material could flow toward the south along the slope of the megadome, whereas the load induced by the flows should accelerate the collapse.

\subsubsection{Collapse controlled by thermal contraction}

The cooling of the residual "warm" ice probably located just beneath Thera Macula may provide a further explanation for the collapse of the megadome. Since density of warm ice is lower than density of cold ice, the cooling of warm ice decreases the volume, producing a contraction and potentially the collapse of the megadome. This added collapse depends on the amount of warm plume material submitted to the cooling. In respect with the extrusion stage, cooling of the warm ice obviously finish the collapse and should close off the dynamical evolution of Thera Macula.

According to morphologic evidences, the upward of a megadome in a recent past seems to have occurred during the emplacement of Thera Macula. However, the deformation of preexisting surfaces to growth a dome as large as about $50 \mathrm{~km}$ in radius is not easy and requires the existence of a positive thermal anomaly just beneath Thera Macula.

Although the evolution of this megadome is poorly constrained, a strong rheological contrast (i.e. a décollement layer) is needed at the base of the upper part of the icy crust to allow the sliding of the blocks. Processes and nature of the shallow material invoked for the doming and collapse of the blocks will be discussed in the following section.

Finally, all types of collapse require the existence of soft material, fluid enough to spread on a large surface toward the south and to explain the deformation implied into the chaotic area. They might act successively to produce this large "hot spot" feature.

\section{Dynamical considerations for the icy crust and comparison with similar features}

On the basis of observations, we have suggested that Thera Macula was resulting from a large-scale uplift of the pre-existing ridged terrains leading to the formation of a megadome more than $50 \mathrm{~km}$ in radius, followed by its collapse. Several forces acting buoyantly in the icy crust may punch the surface on Europa, and bulging can be the result of two major processes. Either buoyant pressure exerted at shallow depth on the upper part of the icy crust builds up a dynamical topography, or temperature variation in the upper part of the icy crust produces a local thermal volumetric expansion of the ice leading to the formation of a dome. That is why we will, in this section, investigate these processes by considering both the diapiric and the cryomagmatic origin for Thera Macula.

\subsection{Liquid injection at shallow depth (Fig. 6a)}


Since observations on Thera Macula suggest the flows of fluid material on Europa's surface, the cryomagmatic origin cannot be excluded. Lithostatic pressure is weak compare to pressure during injection of liquid in a sill-like reservoir in the upper icy crust. Then, morphology of the sill should be closer of a laccolitic shape than a lopolitic shape. Presence of liquid at shallow depth can be caused either by rising of liquid material up to the surface, or by in situ partial melting of crustal material. Nevertheless, we note that in situ melting does not account for the doming since it tends to reduce the volume. Thus, such a process is a consequence but does certainly not drive the setting of Thera Macula.

The existence of a deep ocean beneath the icy crust would imply a hydrostatic imbalance providing a valid mechanism driving the ascent of liquid through the icy crust near the surface (i.e. at a depth $\sim 10 \%$ of the icy crust thickness for pure water). However, rise of liquid up to Europa's surface needs different processes, like volatile-bearing liquid, non-ice substances in solid crust or fluid pressurization of small reservoirs in the icy crust (see Fagents, 2003 for a review). The doming might be the result of local pressure excess by at least two processes: 1) the pressure due to liquid injection and accumulation in a sill-like reservoir and 2) the pressure due to the freezing and crystallization of a fraction of liquid in the sill.

Rising and accumulation of liquid in one or several shallow sills should be accompanied by local increase of temperature. A positive thermal anomaly combined to the presence of nonice contaminants -such as magnesium/sodium hydrates or sulphuric acid detected by NIMS (McCord et al., 1998; Carlson et al., 1999, 2005; Kargel et al., 2001), or ammonia/methane clathrates (Lunine and Stevenson, 1982; Hogenboom et al., 1997; Deschamps and Sotin, 2001; Prieto-Ballesteros et al., 2005)- may allow local partial melting in the icy crust and pressurized reservoirs in the icy crust. Mobilization processes described by Head and Pappalardo (1999) may drive and accumulate these added fluids toward the shallow intrusive layered reservoir(s) and consequently increase the pressure. Partial freezing of the liquid should also lead to a substantial increase in volume and pressure that takes part in the bulging of the surface. Using method for fluid pressurization computation from Fagents (2003, section 3.3.), we calculate that, for a frozen fraction of only $5 \%$ of the initial liquid reservoir and by neglecting the deformation on the reservoir walls, the pressure excess reaches about $10 \mathrm{MPa}$. This value should be large enough to bulge the crust above the reservoir and to propagate cracks up to the surface. The cryomagmatic emplacement of Thera Macula is highly probable since Fagents (2003) has shown that pressure-driven ascent of liquid up to the surface is theoretically possible and easy.

\subsection{Coalescence of "warm" diapirs at shallow depth (Fig. 6b)}

Diapiric intrusion of material up to shallow depth is a common way to explain the formation of various features on Europa (see Pappalardo et al., 1999 for a review). This mechanism was envisaged and modelled for the case of small features like lenticulae (Pappalardo et al., 1998; Rathbun et al., 1998; Tobie et al., 2003), and for Murias Chaos (Figueredo et al., 2002), but application of a simple diapiric rise of ductile "warm" ice to feature as large as Thera Macula is only possible by considering a very thick icy crust (i.e. thickness equivalent or superior to the diameter of the feature), that goes against the results of various works on the external crust of Europa (see Pappalardo et al., 1999). A diapiric origin for Thera Macula is then difficult to envisage. However, Spaun et al. (1998) have proposed an interesting model of coalescence of several diapirs to explain the formation of Conamara Chaos on the basis of observations. This process may also be considered in this case (fig. 6b). A décollement layer may be emplaced at shallow depth by coalescence of several small diapirs and may allow sliding of the blocks. Buoyant pressure exerted by intrusive icy material may significantly raise the upper rigid icy crust to drive the doming. Furthermore, the "warm" geodynamic context beneath Thera Macula may explain the ductile way of the strain and enhance the magnitude of the dome. Density variations induced by a positive thermal 
anomaly play a non-negligible role on the icy crust dynamics. Actually, density of ice decreases as the temperature increases up to the liquidus.

We have evaluated the volume change near the surface due to the density variation in a first order by assuming a purely "cold" conductive icy crust above a "warm" ductile icy crust. Since the diapir reaches the base of the "cold" upper icy crust in one point and that heat diffusion makes one's way towards the three dimensions up to the surface, we will consider an initial spherical portion of the "cold" icy crust that is tangent to the surface as shown in Fig. 7. By supposing that the megadome represents the emergent part of a larger spherical portion corresponding to the dilation of the initial one (dark grey in Fig. 7d) induced by a positive thermal anomaly, the initial radius $R_{i}$ corresponding to the "warming area" of an applied positive thermal anomaly at the base can be computed for various values of "cold" icy crust thickness $b$ and height of the megadome $h$ following:

$$
R_{i}=\sqrt{b\left(\frac{R^{2}-h^{2}}{h} b\right)}
$$

Then, volumes of both initial and final spherical portions can be assessed as following:

$$
\begin{aligned}
& V_{i}=\frac{\pi b^{2}}{3}\left[3 R_{i}-b\right] \\
& V_{f}=\frac{\pi(b+h)^{2}}{3}\left[3\left(R_{i}+h\right)-(b+h)\right]
\end{aligned}
$$

According to the mass conservation principles, the corresponding density ratio that satisfy to these volume changes can be computed as following:

$$
\frac{\rho_{c}}{\rho_{w}}=\frac{V_{f}}{V_{i}}
$$

A Thera's mean radius $R=50 \mathrm{~km}$ has been used here in order to simplify the calculations. By using equation (4.1) to (4.4), the values of the density ratio as a function of the height of the megadome $(h)$ for various "cold" icy crust thicknesses $(b)$ have been plotted in the diagram of figure 8 . The dashed line indicates the density ratio for a mean "cold" ice density $\rho_{c}=932$ $\mathrm{kg} \cdot \mathrm{m}^{-3}$ close to the surface and a "warm" ice density $\rho_{w}=916 \mathrm{~kg} \cdot \mathrm{m}^{-3}$ in depth. The radius of the thermal anomaly at the base of the crust $R_{i}$ decreases quickly when $h$ increases and corresponds to $\sim 535 \mathrm{~km}$ for the ice density ratio given by the dashed line on Fig. 8 . Thicker is the "cold" icy crust and higher is the megadome. The height of the dome only reaches $\sim 17 \mathrm{~m}$ for a "cold" icy crust of $2 \mathrm{~km}$ in thickness, and respectively $~ 86 \mathrm{~m}$ for $10 \mathrm{~km}$. This elevation due to volume changes remains insufficient to explain by itself Thera Macula's formation, but it certainly contribute to the growth of the megadome. Furthermore, these results represent a maximal estimate of the effects of thermal expansion of a "cold" icy crust into a "warm" icy crust since thermal gradients are neglected in the "cold" upper icy crust and since lateral movements of ductile icy material, especially in depth, have not been taken into account. Nevertheless, isostatic effects are also neglected and tend to uplift the surface. For example, a first order calculation of isostatic equilibrium occurring in an upper icy crust (i.e. by neglecting thermal gradients) provides the elevation of the surface $\left(h_{\text {isostatic }}\right)$ as a function of the upper icy crust thickness $(b)$ following $h=b \cdot\left(\rho_{c} / \rho_{w}\right)-b$. This relation gives that a thickness $b=2 \mathrm{~km}$ enhances the doming by $h \sim 35 \mathrm{~m}$. Finally, the main result of these simple calculations is that the thermal expansion of a "cold" icy crust into a "warm" icy crust is nonnegligible, and contribute to drive the growth of a megadome as large as Thera Macula, even if a more important mechanism is required.

\subsection{Is Thera Macula an exception or the rule among Chaos similar in size?}


Although Thera was first classified as a "Macula" (i.e. "Dark spot, may be irregular" as defined by IAU nomenclature) on the basis of Voyager images, the recent medium and high resolution Galileo images suggest that it may, at least partly, belong to "Chaos" family. We will compare Thera with two others large chaotic area well known -because well imagedon Europa: Conamara Chaos $\left(9.7^{\circ} \mathrm{N} \& 272.7^{\circ} \mathrm{W}\right)$ and Murias Chaos $\left(22.4^{\circ} \mathrm{N} \& 83.8^{\circ} \mathrm{W}\right)$. Both Chaos have dimensions and, in places, textures similar to Thera Macula.

These three features have morphologies and textures characteristic of chaotic areas: a dark and rugged matrix embedding blocks of various sizes, sometimes tilted, coming from the disruption of pre-existing ridged terrains (e.g. Collins et al., 2000). They are all relatively young compared to the mean surface age because they are weakly crossed-cut by ridges or cracks, and the crater density on their surface is very weak. At medium resolution, their boundaries appear relatively sharp and show laterally important change of morphology. Matrix material lies often down the surrounding ridged plains, confined by cliffs tens of meters or more. In places, matrix material is more elevated and flows over the ridged plains (see Table 2). We can distinguish sometimes dark and diffuse patches along the external border of chaotic areas (see Table 2) which textures may suggest that fluid material coming from the matrix area was seeped in the ridged plains (Kortz et al., 2000). Only Murias Chaos doesn't exhibit "seepage patch", however rugged and degraded material coming from the matrix overflows onto the ridged plains (Figueredo et al., 2002). On the other hand, Thera and Murias boundaries differ from those of Conamara since they are marked by network of narrow and long fractures. Table 2 reports observations on the three features and clearly shows that even though they locally have their own distinctive features, they are close together. Such similarities bring to questions about the nature of processes occurring in the icy crust to produce these structures: Are the same processes implied for Thera, Murias and Conamara Chaos? We will discuss about this question in the following section.

\section{Discussion and conclusions}

\subsection{Discussion}

Interpretations and investigations presented here lead to propose that both cryomagmatic and diapiric processes may produce a surface megadome as large as Thera Macula. Though the low surface temperature implies a rigid and brittle upper icy crust, observations point out the persistence of a peripheral bulge after the collapse. This is a strong argument for the existence of an irreversible strain. Actually, purely elastic strain would not produce surface excess required to preserve the concentric bulge. Then, processes implied for the formation of Thera's megadome need ductile stretching on the border of the dome (i.e. at the concentric bulge emplacement), and probably up to its centre. Although the surface temperatures are very low, this way of deformation is not paradoxical with rheological surface properties but suggests that strain rates lifting the megadome upward are relatively slow and then processes timescales relatively long.

Thermal expansion of ice may be itself sufficient to finish the growth of a megadome $50 \mathrm{~km}$ in radius. However, our calculations are probably slightly overestimated. 1) Our simple model considers that volume change occurs homogeneously over the whole icy crust up to the surface. Morphologies of the northern area (see paragraph 2.2.) suggest movements but not necessary volume change of the surface layer (e.g. melting is not required). The icy crust should consequently be thicker than expected. Furthermore, we suppose a homogeneous pure icy crust, but contaminants such as clathrate hydrates, magnesium, sodium hydrates or sulphuric acid may be mixed with ice and locally disturb the heat transfer and the densities (Crawford et al., 1988; McCord et al., 1998; Carlson et al., 1999; 2005; Prieto-Ballesteros et al., 2005). 2) The thermal gradient in the "cold” icy crust was neglected. The density contrast 
and then volume change are together reduced, and larger initial ice volumes are required to produce equivalent megadome. 3) The effects of lithostatic pressure were neglected and radial flow of "warm" ice may occur in depth where the temperatures are the most favourable. This could modify the spherical shape of the "warming zone" into a bell shape, that is to say by reducing the height of the megadome and increasing the basal radius of the "warm" part of the icy crust. Although these arguments tend to reduce the contribution of a thermal volume change to the growth of the megadome, thermal processes are strongly non-negligible.

Both cryovolcanism and diapirism imply a stratification of the icy crust beneath Thera Macula. On the one hand, emplacement of a sill system layers the icy crust from both thermomechanical and chemical points of view, even after the total freezing of the reservoir (i.e. depending on the chemical nature of the liquid). On the other hand, accumulation of small diapirs defines a "warm" and enriched with non-ice contaminants layer zone possibly corresponding to the base of the conductive lid in agreement with the Fig. 7a-c.

The high similarity between chaotic features considered in section 4.3. leads to compare the nature of processes occurring in the icy crust to produce them. We suggest to consider that Thera Macula may be an archetype of chaotisation processes, at least at largescale, since a part of it was preserved and not covered by any overflowing material. In this way, insights about early stages of deformation were preserved and particularly the bulge surrounding Thera Macula. Then, the emplacement scenario proposed in this work may be similar to other chaotic structures implying doming, material overflowing and/or infiltrations. We note that Figueredo et al. (2002) also conclude to the existence of a doming stage on Murias Chaos at a slightly smaller scale on the basis of observations and topographic investigations on domes on E17 images. Moreover, such an evolutionary model links domes and lenticulae together at a small-scale (10 to $15 \mathrm{~km}$ in diameter; Pappalardo et al., 1998, 1999; see Fig. 8). Although Conamara Chaos is close to Thera Macula in size, it seems to be difficult to find a link since 1) the existence of a global convexity in the past remains not demonstrated (i.e. no concentric bulge), and 2) the overflows are locally observed but are smaller in extent and volumes than for Thera. Furthermore, Conamara's context is slightly different. Actually, plains surrounding Conamara Chaos are covered in pits, domes and lenticulae. This may attest to different process for chaos formation, such as coalescence of small diapirs (like those responsible for lenticulae formation; see Spaun et al., 1998). These differences may be due, for example, to a thicker icy crust beneath Thera Macula than beneath Conamara Chaos. That is why a question mark covers Conamara in figure 9. In another respect, on the contrary to Thera Macula, all of the area undergone chaotisation process and concentric bulge may have totally been embedded before a global relaxation of Conamara (suggesting that it may be older than Thera or Murias). Besides the small overflows show that matrix material was locally higher than surrounding terrains (see Table 2). In this way, Thera Macula may be an intermediate stage representative of chaotisation processes as shown in figure 9 because of its uncompleted development (i.e. asymmetry and preserved fossil bulge). These processes include: 1) a slow doming stage implying ductile and brittle deformation, 2) a collapse of the dome implying disruption of pre-existing terrains, seepage and overflowing of fluid enough material, and 3) a slow relaxation to readjust the surface at the hydrostatic level. Although this scenario is well-know at small-scale (i.e. applied to lenticulae formation), our work highlights that doming may also occur at largescale (40 to $70 \mathrm{~km}$ in radius) on Europa. The apparent lack of large doming features detected on Europa's surface can be understand as a statistical effect in time due to their strong instability and their fast collapse.

Finally, we note that terrains looking-like ancient chaotic units cover large portions of Europa's surface (Riley et al., 2000). Because of their apparent ancient ages and their difference in size, these units are difficult to study and our definition of "large-scale" structures is limited to the well-defined features described and compared in this work. The larger chaotic units identified by Riley et al. (2000) may result from the stacking of multiple 
large-scale but well-defined chaos following mechanisms described in this work, as well as from different processes.

\subsection{Conclusions}

This note reports the structural description of Thera Macula and presents the arguments to explain its emplacement processes. Although Thera Macula is very similar to other chaotic features (e.g. Conamara or Murias Chaos) and could easily be listed among the Chaos family, it remains exceptional for its asymmetric morphology. Our observations suggest a large-scale endogenic origin for Thera Macula by punching and doming of the preexisting ridged surface over about $50 \mathrm{~km}$ in radius implying ductile stretching on its borders. Even if the main process controlling the growth of the megadome is obviously difficult to identify and depends on the nature of the subsurface material, thermal modifications are of primordial importance. Both coalescence of small clathrates-enriched diapirs or development of a cryomagmatic system are valid mechanisms involving the growth of a megadome implying respectively liquid water or ductile ice at shallow depth, but fluid enough material (e.g. briny slush). Whatever the emplacement type and the origin of the megadome, local changes of the thermal regime -associated to volume changes- and a layered structure of the crust are expected just beneath Thera Macula.

The consecutive collapse of the megadome completes the brittle disruption of preexisting surface and may drive overflowing and/or infiltrations of fluid material through the surrounding plains. Finally, stages of formation of Thera Macula proposed here could be generalized to other large chaotic areas, whose Conamara Chaos, despite its differences, may represent the final stage after relaxation. We propose that processes invoked through literature to explain the formation of smaller sub-circular features (e.g. domes and lenticulae) remain similar to those inferred for Thera Macula. Accurate altimetric information and systematic investigations on well-defined chaotic features as well as on larger and more ancient chaotic areas are obviously needed to attempt to quantify the strain over Thera Macula and to check the validity of our model at "large-scale" (40 to $70 \mathrm{~km}$ in radius) and its reproducibility to larger scales.

\section{Acknowledgments}

Firstly, we thank the reviewers for their precious comments about this manuscript. We also thank Jérôme Weiss, Gabriel Tobie, Jean-Philippe Combes, Pierre Arroucau and JeanBaptiste Aurejac for their helpful discussions, which have contributed to improve this study. This work was supported by the INSU Programme National de Planétologie.

\section{References}

Carlson, R.W., Johnson, R.E., Anderson, M.S., 1999. Sulfuric acid on Europa and the radiolytic sulfur cycle. Science 286, 97-99.

Carlson, R.W., Anderson, M.S., Mehlman, R., Johnson, R.E., 2005. Distribution of hydrate on Europa: further evidence for sulfuric acid hydrate. Icarus 177, 461-471.

Cassen, P., Reynolds, R.T., Peale, S.J., 1979. Is there liquid water on Europa? Geophys. Res. Lett. 6, 731-734.

Collins, G.C., Head, J.W., Pappalardo, R.T., Spaun, N.A., 2000. Evaluation of models for the formation of chaotic terrain on Europa. J. Geophys. Res. 105, E1, 1709-1716. 
Crawford, G.D., Stevenson, D.J., 1988. Gas-driven water volcanism and the resurfacing of Europa. Icarus 73, 66-79.

Deschamps, F., Sotin, C., 2001. Thermal convection in the outer shell of large icy satellite. J. Geophys. Res. 106, 5107-5121.

Fagents, S.A., 2003. Considerations for effusive cryovolcanism on Europa: the post-Galileo perspective. J. Geophys. Res. 108, E12, 5139.

Figueredo, P.H., Greeley, R., 2000. Geologic mapping of the northern leading hemisphere of Europa from Galileo solid-state imaging data. J. Geophys. Res. 105, E9, 22629-22646.

Figueredo, P.H., Chuang, F.C., Rathbun, J., Kirk, R.L., Greeley, R., 2002. Geology and origin of Europa’s “Mitten” feature (Murias Chaos). J. Geophys. Res. 107, E5, 2 1-15.

Greeley, R., Collins, G.C., Spaun, N.A., Sullivan, R.J., Moore, J.M., Senske, D.A., Tufts, B.R., Johnson, T.V., Belton, M.J.S., Tanaka, K.L., 2000. Geologic mapping of Europa. J. Geophys. Res. 105, E9, 22559-22578.

Greenberg, R., Leake, M.A., Hoppa, G.V., Tufts, B.R., 2003. Pits and uplifts on Europa. Icarus 161, 102-126.

Head, J.W., Pappalardo, R.T., 1999. Brine mobilization during lithospheric heating on Europa: implications for formation of chaos terrain, lenticula texture and color variations. J. Geophys. Res. 104, 27143-27155.

Hogenboom, D.L., Kargel, J.S., Consolmagno, G.J., Holden, T.C., Lee, L., Buyyounouski, M., 1997. The ammonia-water system and the chemical differentiation of icy satellites. Icarus 128, 171-180.

Kargel, J.S., Head, J.W., Hogenboom, D.L., Khurana, K.K., Marion, G., 2001. The system sulphuric acid - magnesium sulfate - water: Europa's ocean properties related to thermal state. In: Proc. Lunar Planet. Sci. Conf. $32^{\text {st }}$. Lunar and Planetary Institute, Houston, Texas. Abstract \#2138 [CD-ROM].

Kortz, B., Head, J.W., Pappalardo, R.T., 2000. Thrace Macula, Europa: characteristics of the southern margin and relations to background plains and Libya Linea. In: Proc. Lunar Planet. Sci. Conf. $31^{\text {st }}$. Lunar and Planetary Institute, Houston, Texas. Abstract \#2052 [CD-ROM].

Lucchitta, B.K., Soderblom, L.A., 1982. The geology of Europa. In: Morrison, D., (Eds.), Satellites of Jupiter, University of Arizona Press, Tucson, pp. 521-555.

Lunine, J.I., Stevenson, D.J., 1982. Formation of the Galilean satellites in a gaseous nebula. Icarus 52, 14-39.

McCord, T.B., Hansen, G., Fanale, F., Carlson, R., Matson, D., Johnson, T., Smythe, W., Crowley, J., Martin, P., Ocampo, A., Hibbitts, C., Granahan, J., and the NIMS Team, 1998. Salts on Europa's surface detected by Galileo’s Near Infrared Mapping Spectrometer. Science 280, 1242-1245.

McKinnon, W.B., 1999. Convective instability in Europa's floating ice shell. Geophys. Res. Lett. 26, 951-954. 
Mével, L., Mercier, E., 2005. Resorption process in Astypalaea Linea extensive region (Europa). Planetary and Space Science 53, 771-779.

Ojakangas, G.W., Stevenson, D.J., 1989. Thermal state of an ice shell on Europa. Icarus 81, 220-241.

Pappalardo, R.T., Head, J.W., Greeley, R., Sullivan, R.J., Pilcher, C., Schubert, G., Moore, W.B., Carr, M.H., Moore, J.M., Belton, M.J.S., 1998. Geological evidence for solid-state convection in Europa's ice shell. Nature 391, 365-368.

Pappalardo, R.T., Belton, M.J.S., Breneman, H.H., Carr, M.H., Chapman, C.R., Collins, G.C., Denk, T., Fagents, S., Geissler, P.E., Giese, B., Greeley, R., Greenberg, R., Head, J.W., Helfenstein, P., Hoppa, G., Kadel, S.D., Klaasen, K.P., Klemaszewski, J.E., Magee, K., McEwen, A.S., Moore, J.M., Moore, W.B., Neukum, G., Phillips, C.B., Prockter, L.M., Schubert, G., Senske, D.A., Sullivan, R.J., Tufts, B.R., Turtle, E.P., Wagner, R., Williams, K.K., 1999. Does Europa have a subsurface ocean ? Evaluation of the geological evidence. J. Geophys. Res. 104, E10, 24015-24056.

Prieto-Ballesteros, O., Kargel, J.S., Fernandez-Sampedro, M., Selsis, F., Martinez, E.S., Hogenboom, D.L., 2005. Evaluation of the possible presence of clathrate hydrates in Europa’s icy shell or seafloor. Icarus 177, 491-505.

Rathbun, J.A., Musser, G.S., Squyres, S.W., 1998. Ice diapirs on Europa: implications for liquid water. Geophys. Res. Lett. 25, 4157-4160.

Reynolds, R. T., Cassen, P.M., 1979. On the internal structure of major satellites of the outer planets. Geophys. Res. Lett. 6, 121-124.

Riley, J., Hoppa, G.V., Greenberg, R., Tufts, B.R., and Geissler, P., 2000. Distribution of chaotic terrain on Europa. J. Geophys. Res. 105, E9, 22599-22615.

Spaun, N.A., Head, J.W., Collins, G.C., Prockter, L.M., Pappalardo, R.T., 1998. Conamara Chaos region, Europa: reconstruction of mobile polygonal ice blocks. Geophys. Res. Lett. 25, 4277-4280.

Thomson, R.E., Delaney, J.R., 2001. Evidence for a weakly stratified europan ocean sustained by seafloor heat flux. J. Geophys. Res. 106, E6, 12355-12365.

Tobie, G., Choblet, G., Sotin, C., 2003. Tidally heated convection: constraints on Europa's ice shell thickness. J. Geophys. Res. 108, E11, 10-1.

Wilson, L., Head, J.W., Pappalardo, R.T., 1997. Eruption of lava flows in Europa: theory and application to Thrace Macula. J. Geophys. Res. 102, 9263-9272.

Zahnle, K.L., Schenk, P., Levison, H.F., Dones, L., 2003. Cratering rates in the outer solar system. Icarus 163, 263-289. 


\section{Figures}
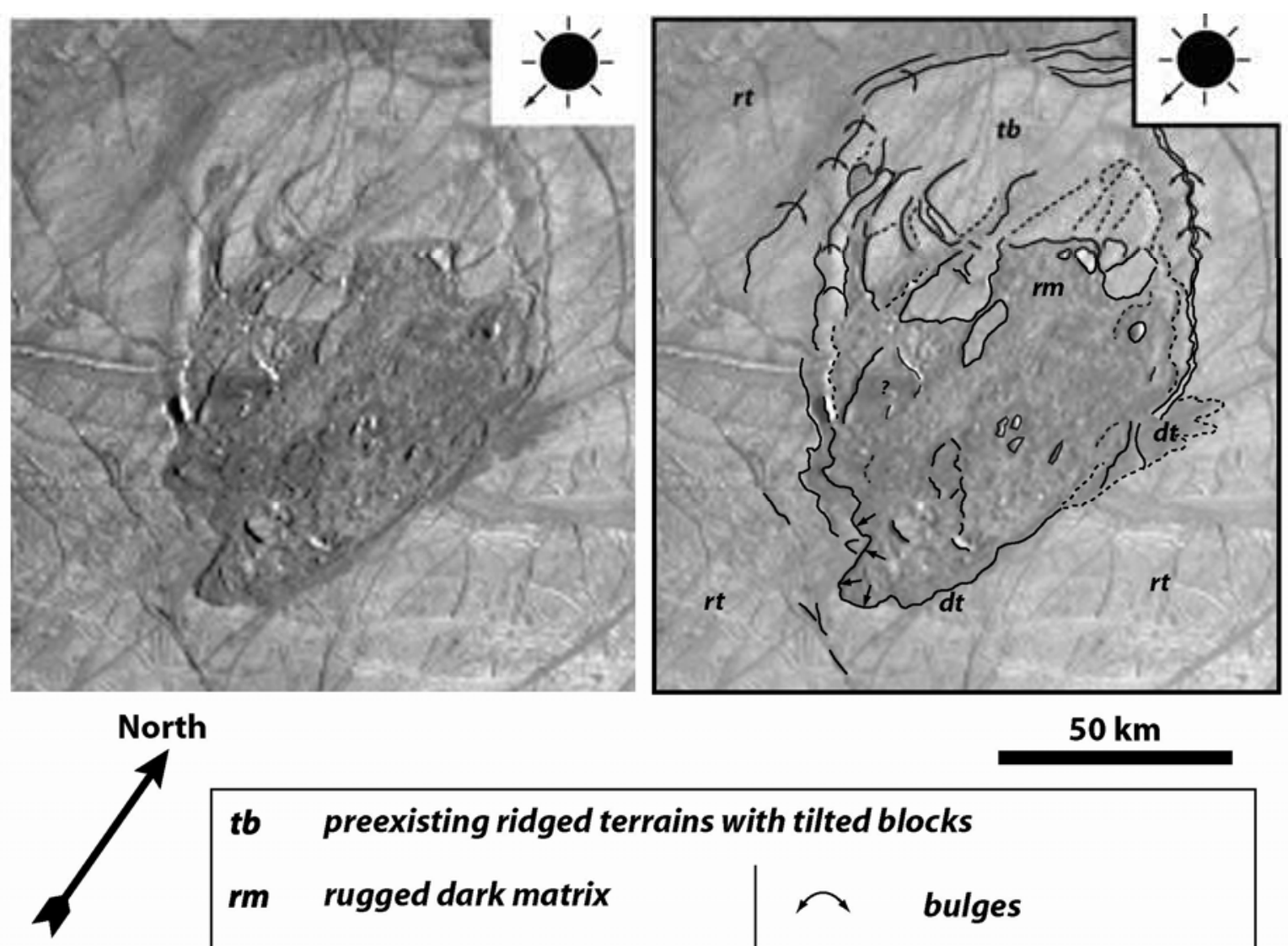

North

$50 \mathrm{~km}$

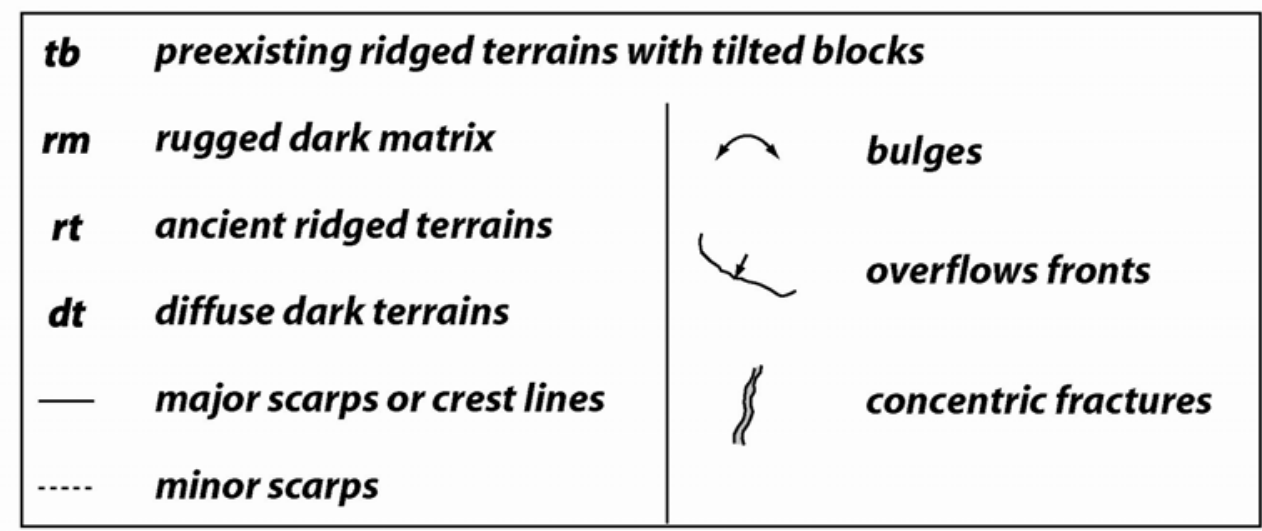

Figure 1

Mével and Mercier

Fig. 1

Morpho-structural map of Thera Macula. Its subcircular slightly elongated shape and its strong asymmetry are well distinguishable on this medium resolution image ( $\sim 300 \mathrm{~m} / \mathrm{pixel})$ acquired during the Galileo's E17 orbit (images 17E0019 and 17E0020). The image is centred at $47.3^{\circ} \mathrm{S}$ and $185.0^{\circ} \mathrm{W}$.

Fig. 2

Details observed inside the chaotic matrix. (a) Cliffs surrounding the matrix and defining the boundary between northern bright area and southern chaotic unit. The concentric bulge rounding Thera Macula is particularly well defined in the west (b) and in the northeast (c) borders. The N-E bulge is characterized by a median sublinear tensile crack at its top (c) that results from the collapse of multi-kilometric blocks ( $d$, white arrows highlight the fracture 
network) through the centre of Thera Macula. Several tilted blocks of pre-existing terrains are visible in the chaotic area ( $e, f$ and $g$ ) and their heights have been reported in table 1 . Finally, a cliff of about $437 \mathrm{~m}$ in height looks down over the bright-ridged plains at the south tip of Thera $(h)$. This cliff suggests the flows of material through the south (white arrow gives the direction of flows). All the scale bars are $10 \mathrm{~km}$ in length. 


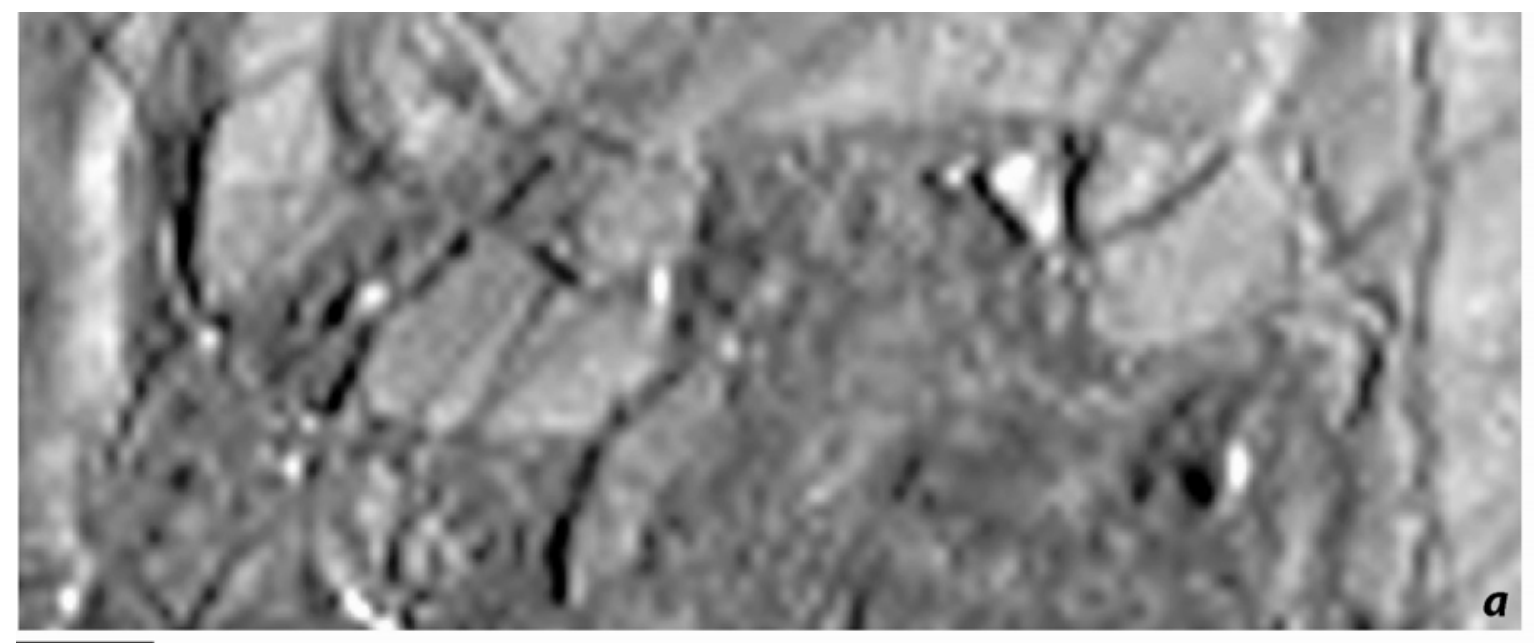

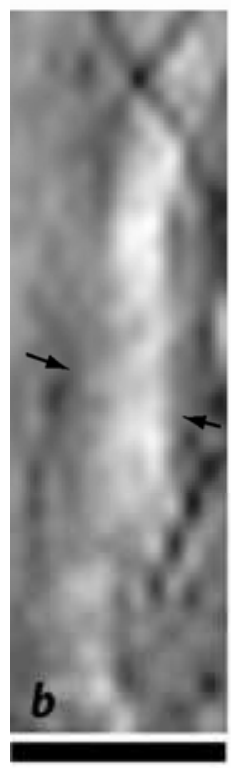
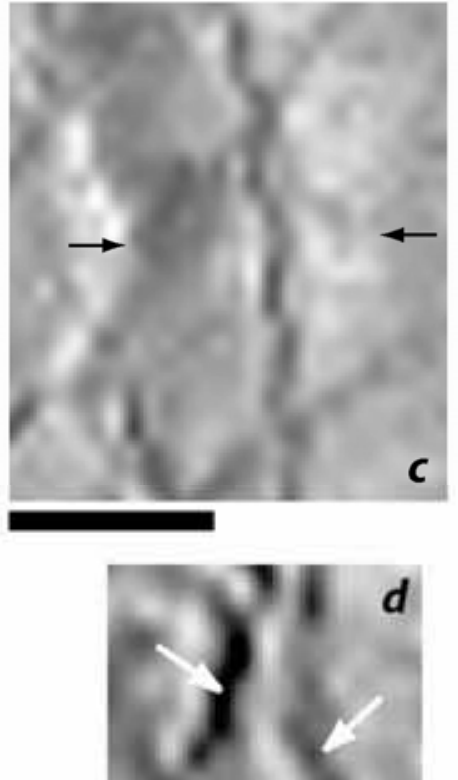

\section{5 metres}

e

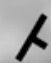

370 metres

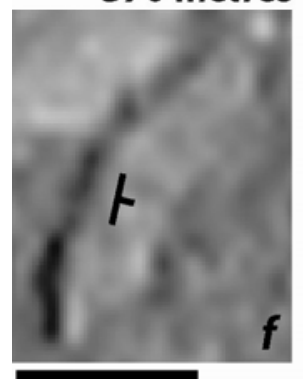

437 metres
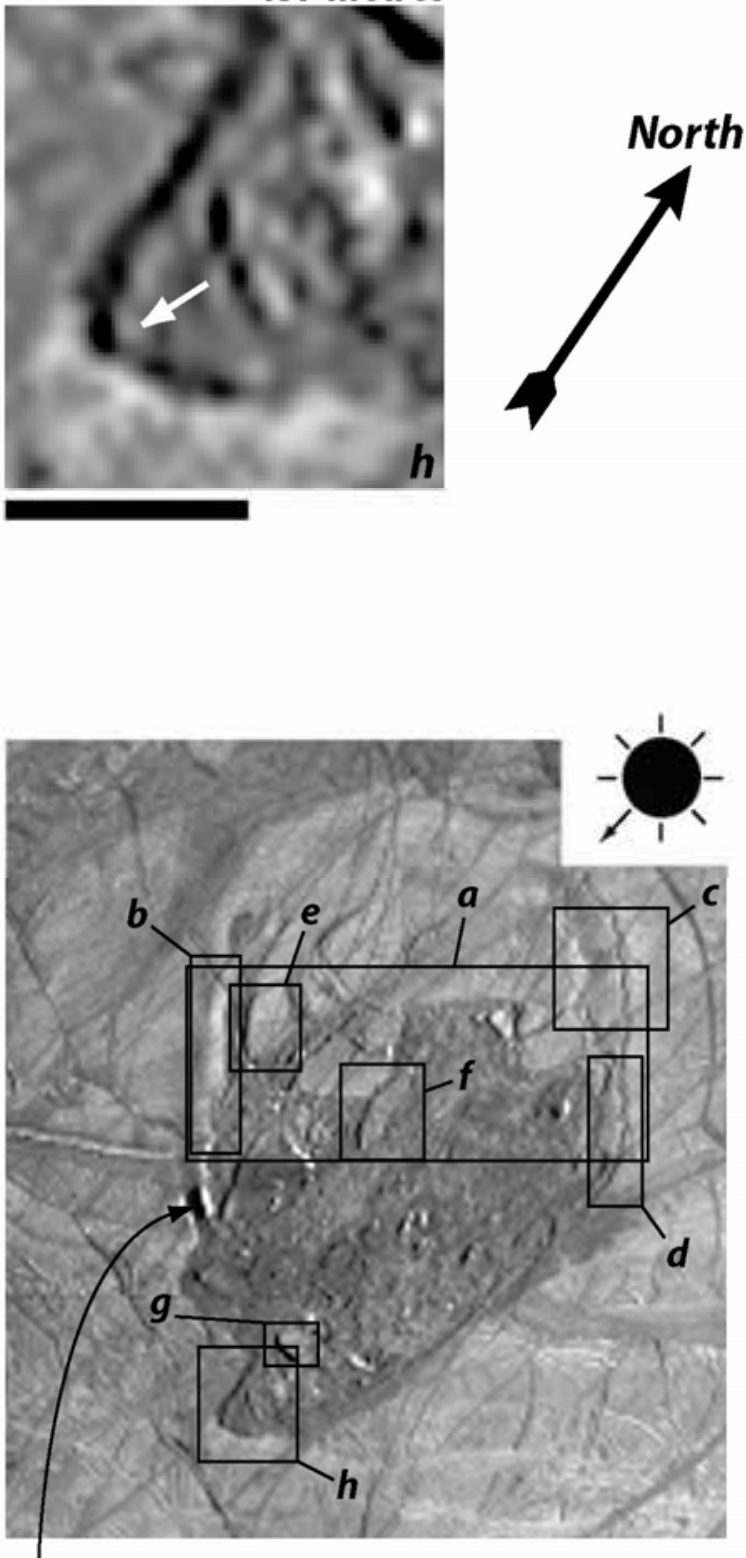

top of Thera 920 metres 


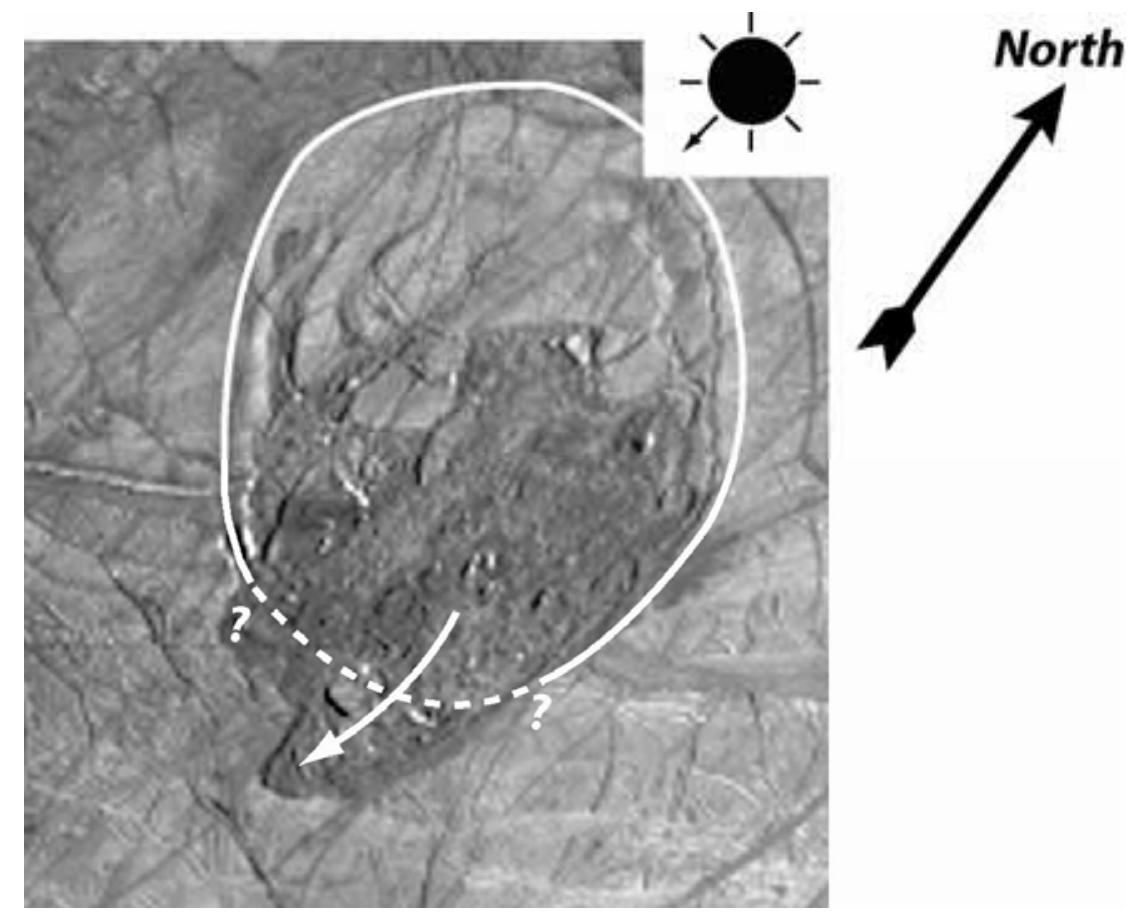

Fig. 3

Matrix material has only overflow through the south border of Thera Macula. Although its recent elongated shape, we believe that its original shape before the overflows of material toward the south was probably defined by the white dashed curve.

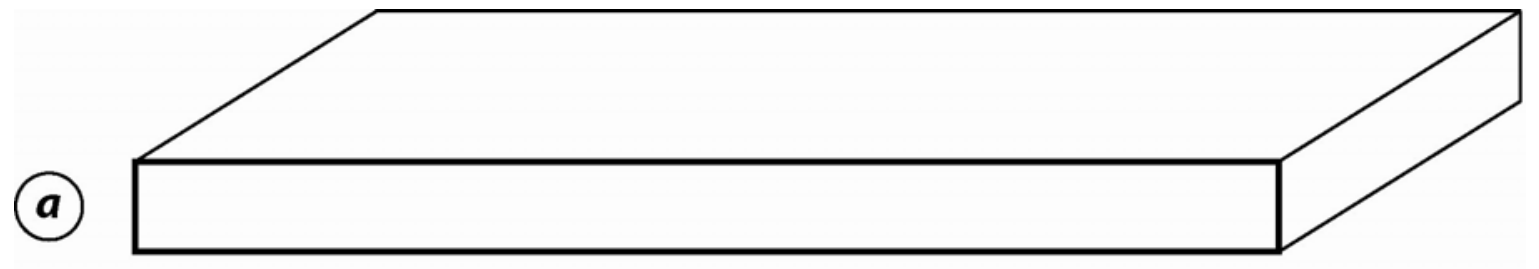

Stretching, crustal thinning and surface increase

(b)

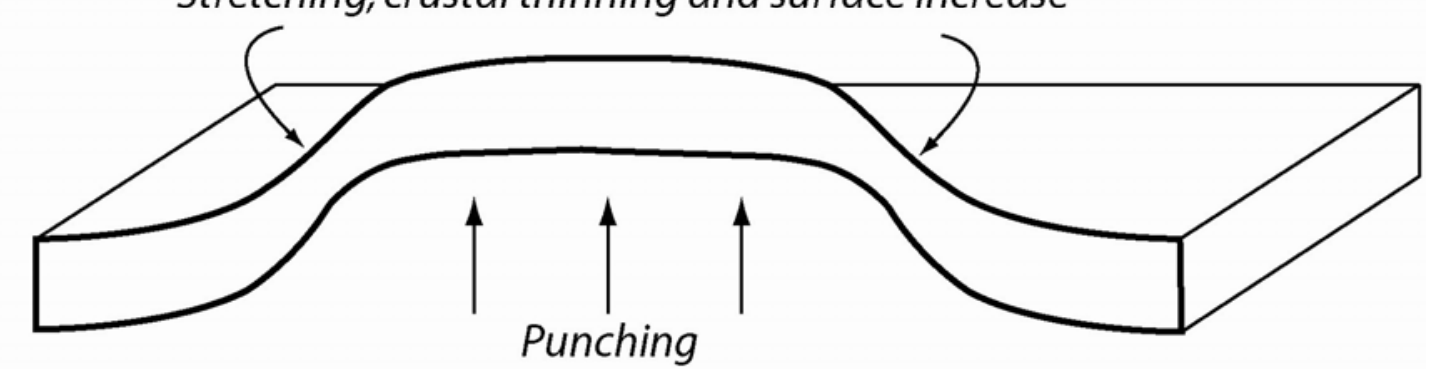

(c)

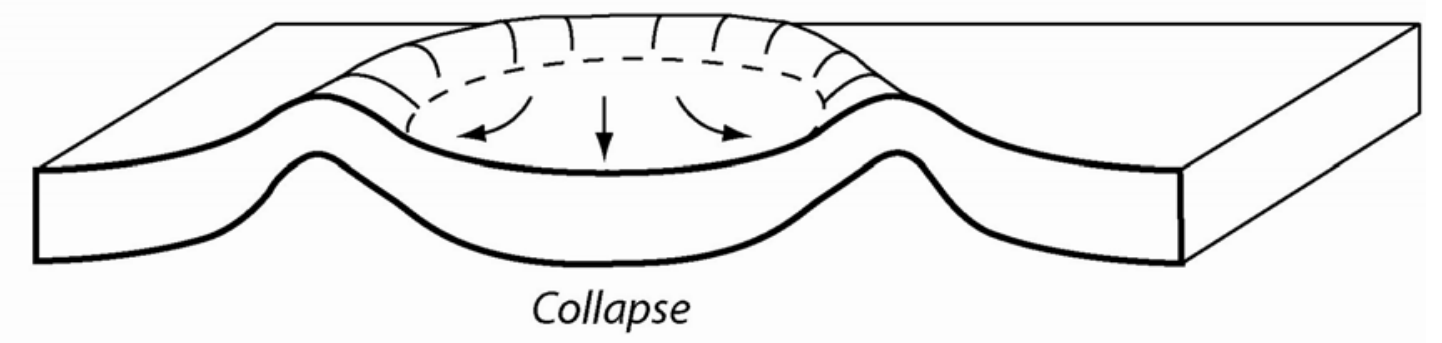

Fig. 4 
Sketches showing the effects of punching of the upper part of the icy crust and creation of a remnant bulge due to ductile stretch. (a) Initial state. (b) Surface uplift by punching implying stretching and crustal thinning on the borders of the dome. (c) Collapse of the dome. Ductile deformation in the stretched area leads to surface excess that produces a peripheral bulge.

(a) South

North

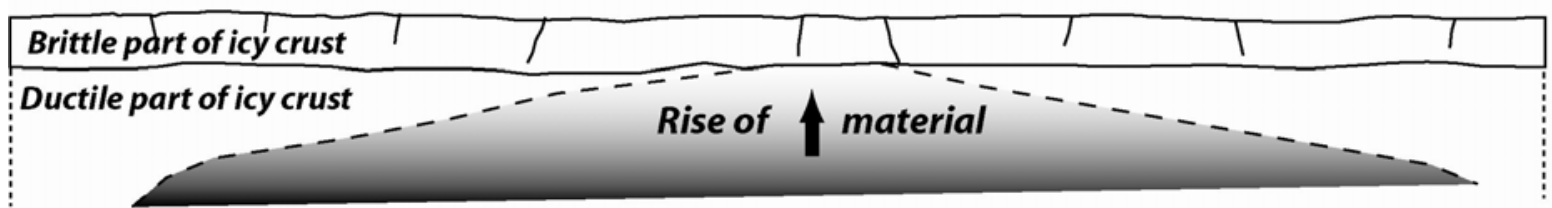

(b)
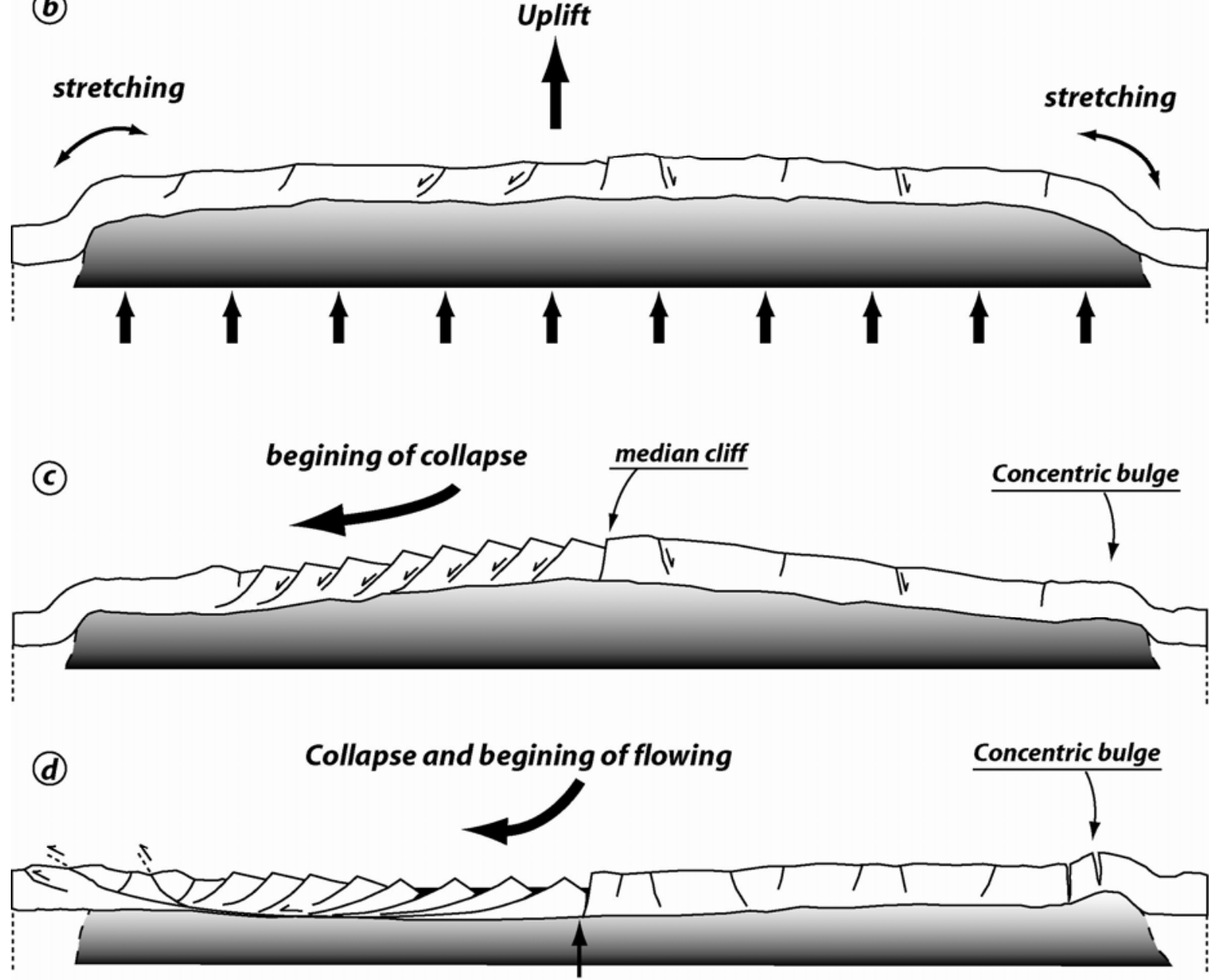

(e) chaotic matrix

northern bright area

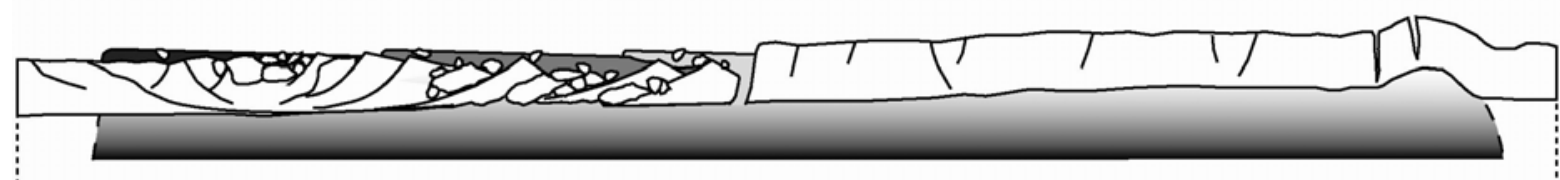

Fig. 5 
Model of evolution of Thera Macula based on structural and morphological observations. (a) "Warm" material rises up to shallow depth and the pressure increases under the rigid upper part of the icy crust. (b) The pressure exerted by the material bulges the superficial part of the icy crust over a radius of about $50 \mathrm{~km}$. Tensile stress on the megadome opens many fractures that define tilted blocks. At the top of the megadome, where the tensile stress reaches its maximal value, a large median fracture appears. This main crack allows the gravity collapse of blocks overlying a décollement layer $(c)$. During the collapse, viscous and/or liquid material flows toward the south $(d-e)$, disrupting the large tilted blocks into smaller blocks. Spreading of "cryoclastic" material may be relatively instantaneous accompanied by explosive disruption of pre-existing terrains according to the Fagents (2003) mechanism. Finally, the collapse may be enhanced either by the early emptying of a cryomagmatic reservoir, or by the late cooling of the warm ice.

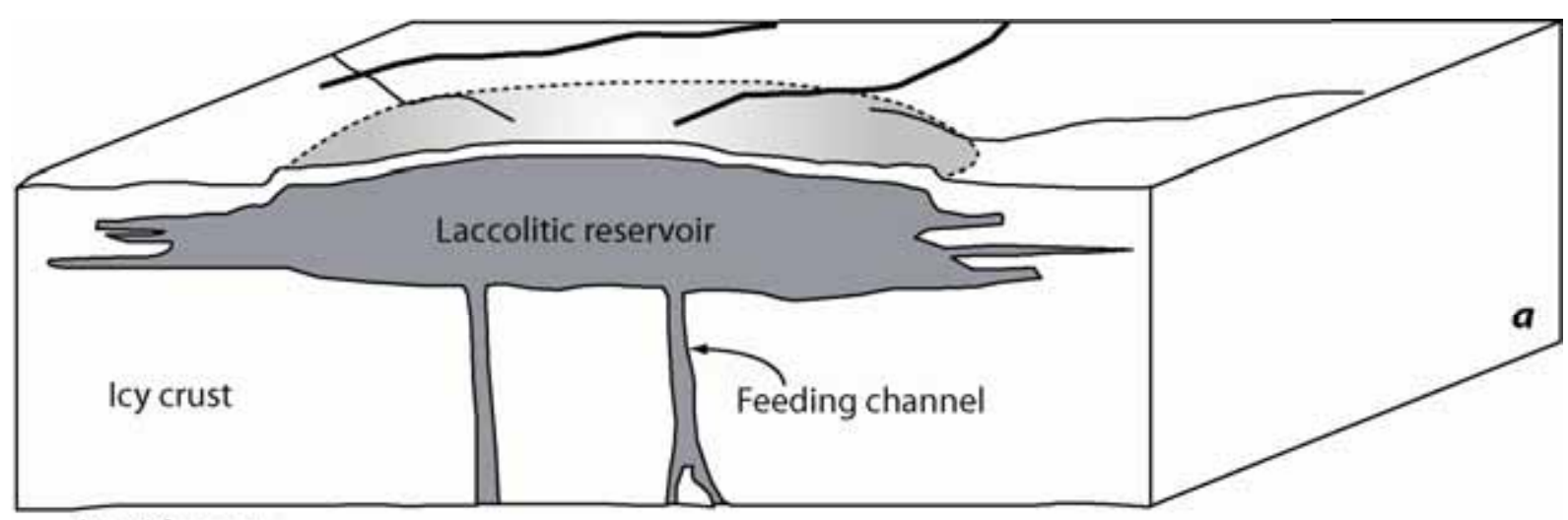

Liquid ocean

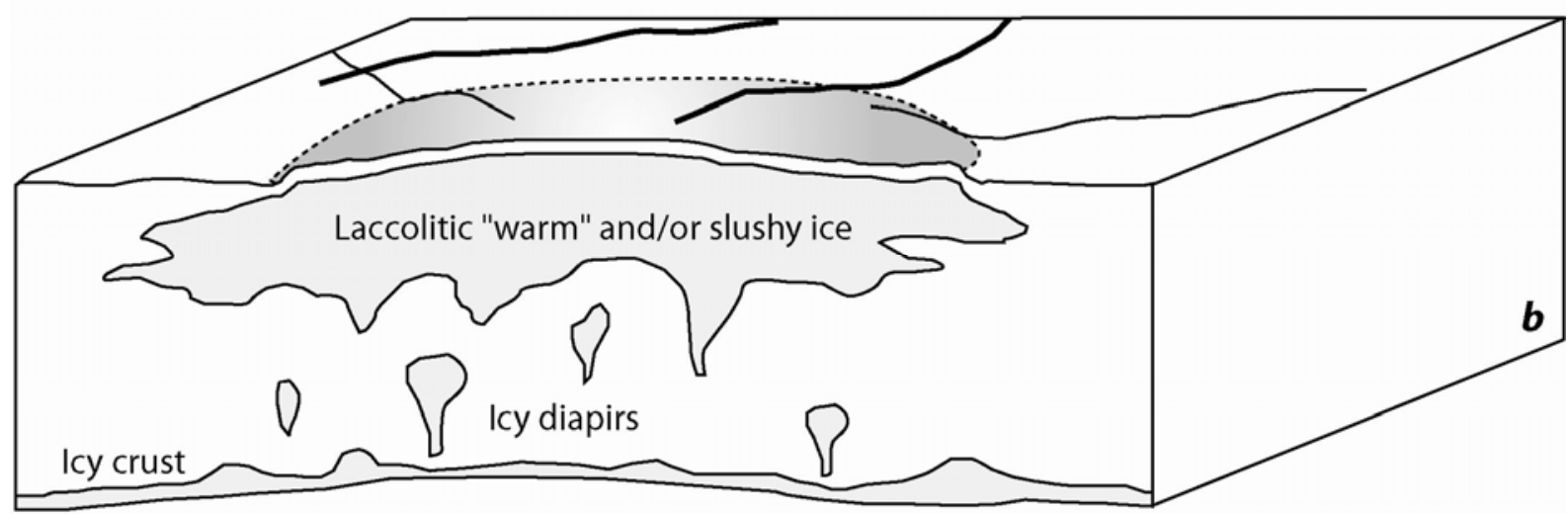

Liquid ocean

\section{Fig. 6}

Cross-sections showing both cryomagmatic $(a)$ and diapiric $(b)$ processes supposed to punch the surface and drive the doming stage beneath Thera Macula (see text for details). 


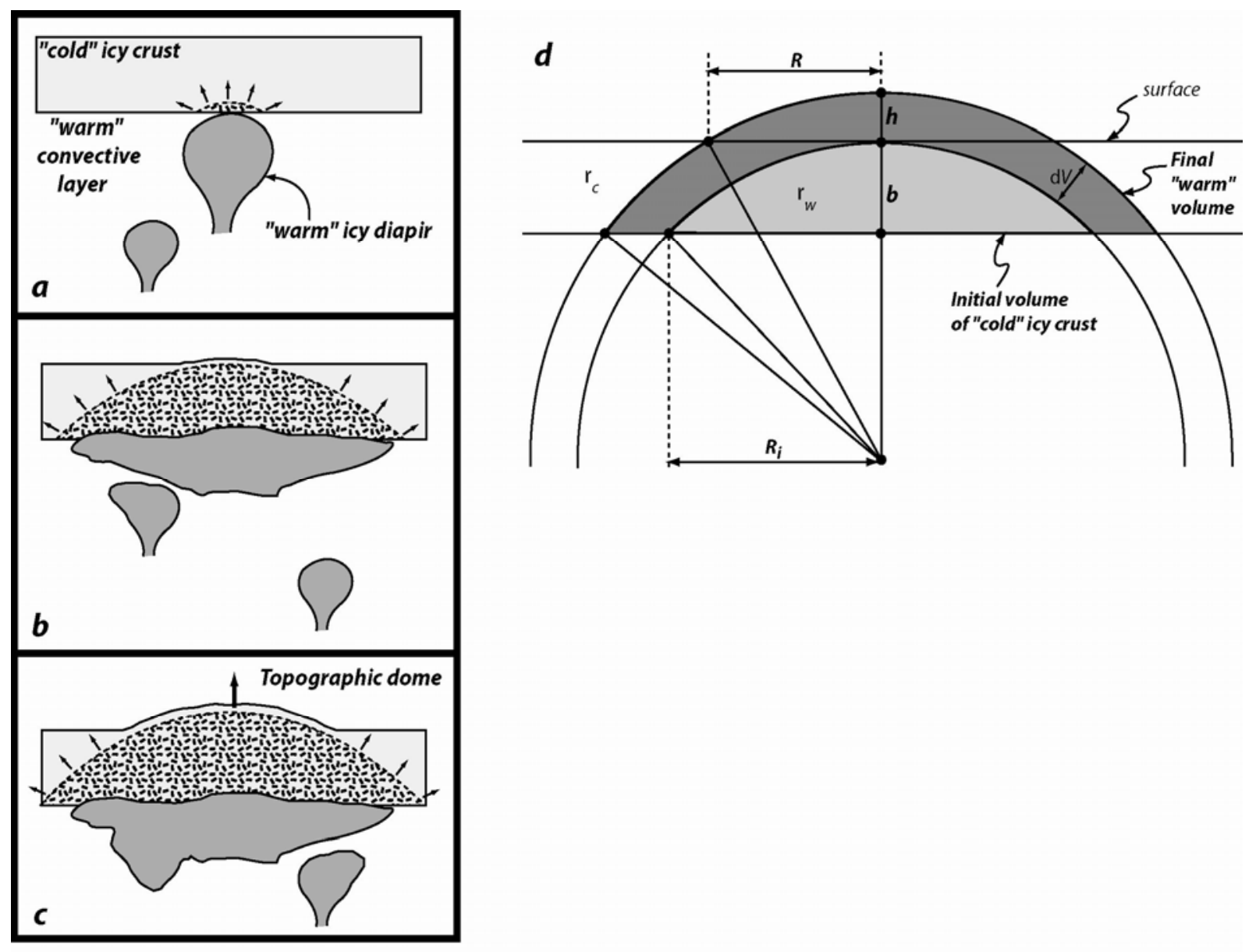

Fig. 7

Sketches illustrating the effects of volume changes occurring in the upper icy crust. (a) "Warm" icy diapirs reach a shallow interface (e.g. the base of the conductive lid) and piled down the "cold" icy crust (b). Heat of the clustered diapirs is transferred by conduction up to the surface and changes the density (and the volume) of the warmed portion of the initial "cold" icy crust. Then, a topographic dome uplifts by volume change $(c)$. Sketch $(d)$ shows the geometrical model used to estimate the contribution of thermal expansion of ice due to a positive thermal anomaly at the base of an icy crust $b$ in thickness to the growth of a megadome $h$ in maximal height. The light-greyed "cold" initial volume becomes the larger dark-greyed "warm" final volume. The volume increase $\delta V$ builds up a volume excess above the initial surface that corresponds to the dome. $R$ is the mean radius of Thera Macula (that is assumed to be $\sim 50 \mathrm{~km}$ ), whereas $R_{i}$ might represent the basal extension of the clustered diapirs. 


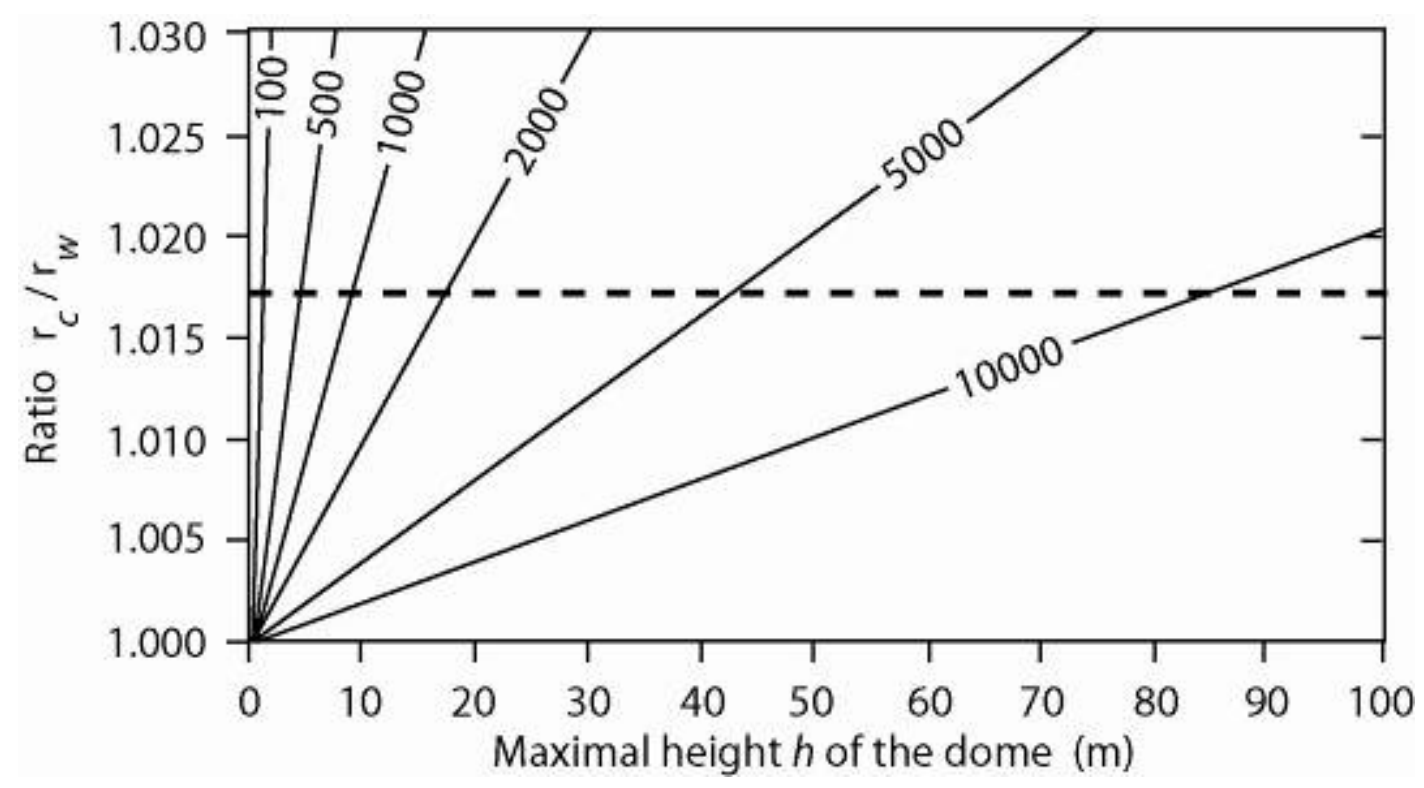

Fig. 8

Density ratio satisfying the crustal geometry described in the Fig. 7 have been plotted as a function of the height of the megadome $(h)$ for "cold" icy crust thicknesses $(b)$ ranging from $100 \mathrm{~m}$ to $10 \mathrm{~km}$. The dashed line indicates the density ratio for a mean "cold" ice density $\rho_{c}=$ $932 \mathrm{~kg} \cdot \mathrm{m}^{-3}$ close to the surface and a "warm” ice density $\rho_{w}=916 \mathrm{~kg} \cdot \mathrm{m}^{-3}$ in depth. These elevations remain probably too low to explain the observed deformations in Thera Macula. This strongly suggests that other processes are needed to punch the surface upward. 


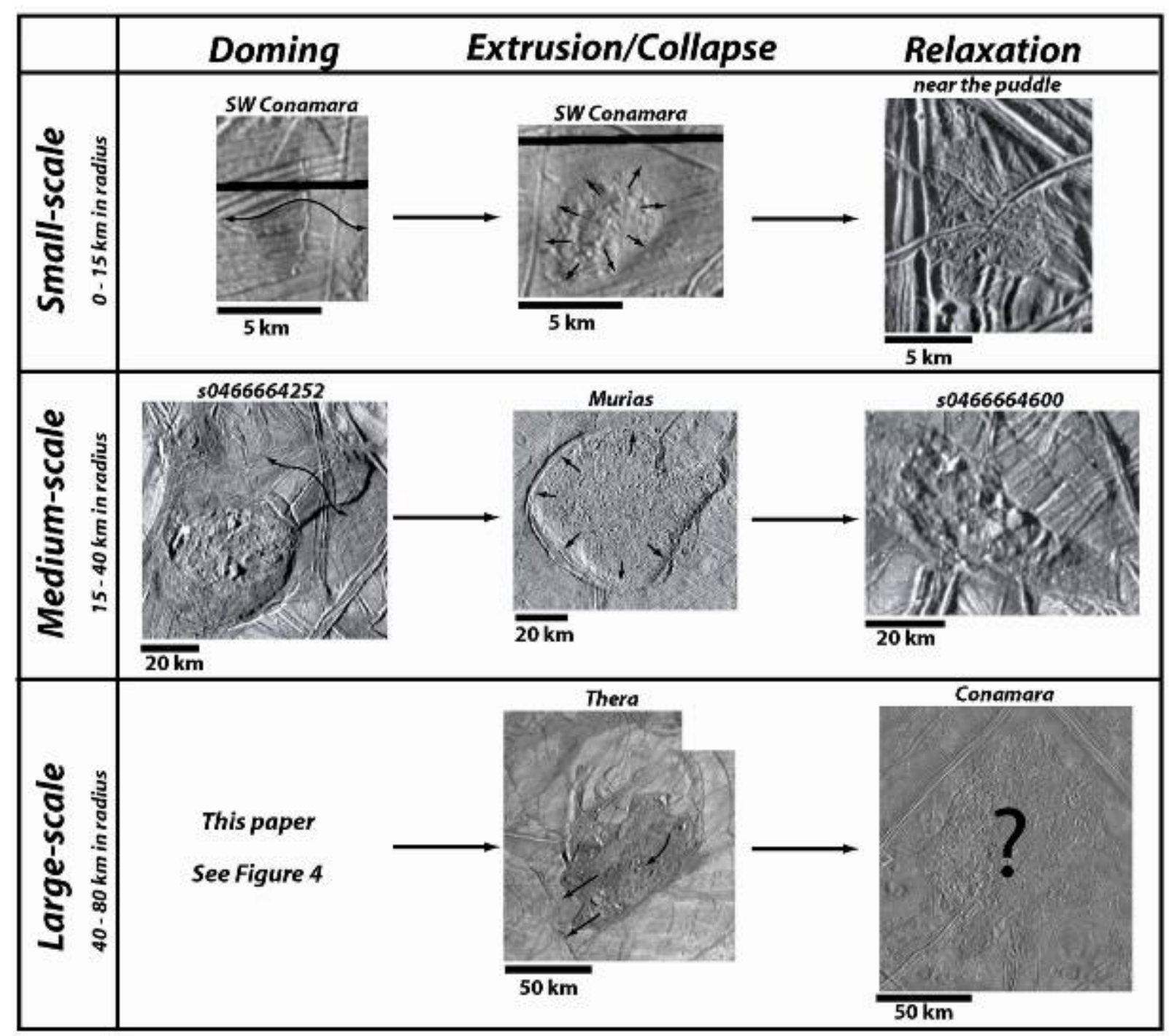

Fig. 9

Synthetic table illustrating the recurrence of the three major stages controlling "hot spot" evolution with time (doming, extrusion/collapse, relaxation) at various scales. Size range of each category is partially arbitrary, however we have used histogram abundance/radius of domes and lenticulae in Conamara Chaos region from Rathbun et al. (1998) for the smallest class, and we have placed Murias Chaos and Thera Macula/Conamara Chaos in separate class in order to respect the conclusions of Figueredo et al. (2002) (i.e. Murias may be the result of an unique large diapir on the contrary to Conamara). We note that no large-scale doming stage (i.e. $40-80 \mathrm{~km}$ in radius) was found on Galileo images. Finally, the link between Thera and Conamara is not so evident through the only observations, as highlights by the question mark. 


\begin{tabular}{|c|c|c|}
\hline observations & heights (m) & Variations (\%) \\
\hline$e$ & 444 & \pm 16.7 \\
\hline$f$ & 370 & \pm 15.4 \\
\hline$g$ & 477 & \pm 16.7 \\
\hline$h$ & 437 & \pm 20.0 \\
\hline
\end{tabular}

\section{Table 1}

Height of some scarps derived from their shadows by using equation 2.1. Letters in the first column report to observations shown in figure 2. The "variations" column corresponds to the gap between maximal and minimal projections of the shadows with respect to the sunlight axis.

\begin{tabular}{|c|c|c|c|}
\hline Observations & $\begin{array}{c}\text { Thera Macula } \\
\text { (This work) }\end{array}$ & $\begin{array}{c}\text { Murias Chaos } \\
\text { (Figueredo et al., 2002) }\end{array}$ & $\begin{array}{c}\text { Conamara Chaos } \\
\text { (Spaun et al., 1998, } \\
\text { Collins et al., 2000) }\end{array}$ \\
\hline Asymmetry & yes & no & no \\
\hline $\begin{array}{l}\text { Matrix } \\
\text { (elevation compared } \\
\text { to the ridged plains) }\end{array}$ & $\begin{array}{l}\sim 50 \% \text { of the surface } \\
\text { (more elevated to the SE } \\
\text { and lower to the NW) }\end{array}$ & $\begin{array}{l}\sim 100 \% \text { of the surface } \\
\quad \text { (more elevated) }\end{array}$ & $\begin{array}{l}\sim 60 \% \text { of the surface } \\
\text { (lower, except locally) }\end{array}$ \\
\hline Preserved blocks & $\begin{array}{l}\text { Few in the matrix, various } \\
\text { size }\end{array}$ & $\begin{array}{l}\text { Very little blocks weakly } \\
\text { preserved }\end{array}$ & $\begin{array}{l}\text { Very various size, lot of } \\
\text { preserved blocks }\end{array}$ \\
\hline Borders & $\begin{array}{l}\text { Concentric bulge, } \\
\text { fractures and cliffs }\end{array}$ & Fractures and cliffs & Cliffs and several fractures \\
\hline $\begin{array}{l}\text { Overflowing (O) and } \\
\text { infiltrations }(I)\end{array}$ & $\begin{array}{c}\text { Many } O \\
I \text { in two places }\end{array}$ & $\begin{array}{l}\text { Many } O \\
\text { No } I\end{array}$ & $\begin{array}{l}\text { Some little } O \\
I \text { limited }\end{array}$ \\
\hline General bulge & yes & yes & Probably not \\
\hline
\end{tabular}

\section{Table 2}

Comparison of observations between three similar features on Europa. 